\title{
Comparing System- and Test Model with Integrated Software-based Signal Simulation
}

\author{
Andreas Kurtz ${ }^{1}$, Bernhard Bauer ${ }^{2}$ and Marcel Koeberl ${ }^{1}$ \\ ${ }^{1}$ BMW Group, 'Integration Electric/Electronics, Software', Knorrstr. 147, 80788 Munich, Germany \\ ${ }^{2}$ Software Methodologies for Distributed Systems, University of Augsburg, Universitaetsstr. 14, 86135 Augsburg, Germany
}

Keywords: $\quad$ Automotive, AUTOSAR, Distributed Systems, Method, Model Based Testing, Path Detection, Path Reduction, Simulation, System Model, Test Automation, Test Model, Testing.

Abstract: $\quad$ Test automation in distributed systems requires new methods in signal simulation for the stimulation of the distributed system. Increasing complexity of electric electronic (E/E) systems enhances the testing-effort. The main challenge is reducing the time consuming manual stimulation in consideration of improving the quality of testing. Currently used systems for test automation with a software-based approach have to be adapted to each hardware and software version of the system to be tested. The approach represented shows a new approach through the integration of a simulation service in the AUTOSAR software architecture. By integrating a generic software-based simulation module with an interaction point at the basic software driver layer, the execution of tests can be automated and improved under consideration of adaptivity and reproducibility.

\section{INTRODUCTION}

Mastering complexity and customer orientation are challenges in the development of electric and electronic $(\mathrm{E} / \mathrm{E})$ functions in the automotive industry. In current and future vehicles, the increase of the distribution of functions and the networking demands new ways of automation for testing customer features. Software bugs are the main reason for malfunctions in new developed cars. In the automotive industry, the safety requirements are outstandingly important because of its impacts. Therefore, there is a need to err on the side of caution.

In this paper, we focus on developing a method for generic signal simulation for testing the system model at total system platform. System model stands for the total system deemed to be a distributed system with its networked hardware (HW) and software components (SWC). The long-term goal is an integrated distributed signal simulation to realize the virtualisation of the customer input for testing the maximum chain of reaction of customer features.

\subsection{Problem Statement}

Increasing complexity of developed functions with shorter developing time leads to exceeding use of methods. Figure 1 shows the raising input of using methods when reducing development time to handle equal effort. In addition, of an increase of system complexity the conventional methods have to change to virtual development methods. Virtualization is a key factor in testing the total system to be able to handle the increase of complexity.

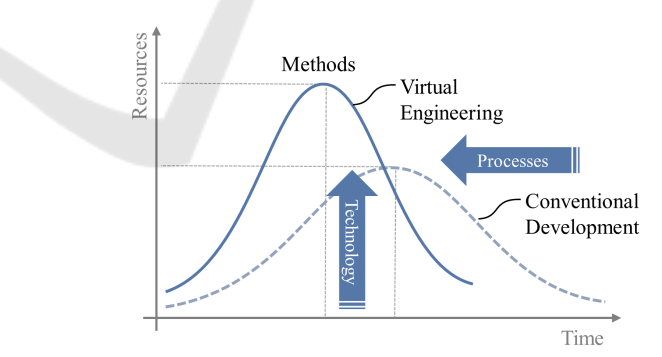

Figure 1: Reducing time demands increase of using methods to manage the effort. (Eigner and Stelzer, 2013).

State-of-the-art integrated simulation functions, used in automotive software development, do set signal values via additional functions integrated in the software components to be tested. If we take a closer look on the kind of implementation, we recognize that the point of interaction is often housed in the implementation of the customer- or a part function. These simulation functions interact with the customer functions and require specific solutions for each type of implementation. 
Using this kind of simulation/testing functions does not reflect the functional behaviour of the software function like in customer usage. Additional signals and interfaces are used to get access to the implemented customer functions.

Another aspect is the additional software code, implemented in the SWC itself, which is needed to realize the interaction. Due to high level of interaction, crosslinked functions are not recognized, so malfunctions in close-by or networked SWCs are not noticed.

Testing by physical simulation of sensor signals at the hardware interface does represent the customer usage but is too complex to find specific solutions for each HW variation. The main challenge for physical signal simulation is to find a generic solution for all types of sensors and interfaces.

Getting a picture of the developed method, we are thinking of a distributed software-based virtualisation of signals in the distributed system. With the focus on the automotive domain, the system architecture is based on AUTOSAR. The generic architecture, of an electronic control unit (ECU), is shown in Figure 2 (AUTOSAR Partnership, 2014).
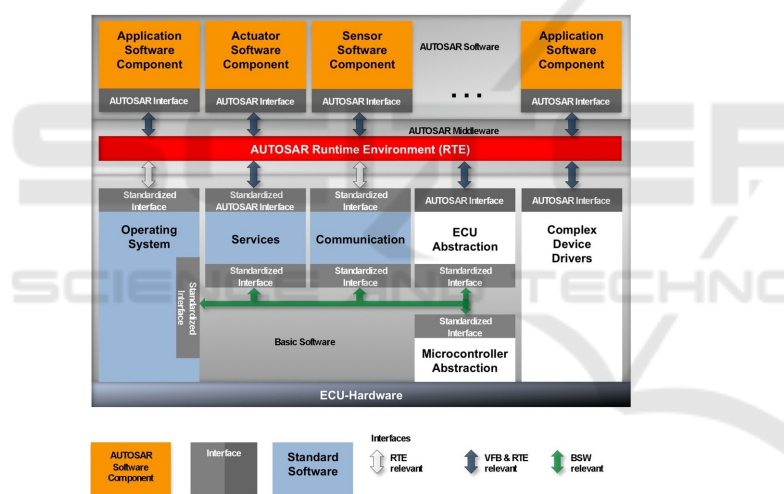

Figure 2: AUTOSAR Architecture, Components and Integration (AUTOSAR Partnership, 2014).

\subsection{State-of-the-Art}

Established methods in model based testing, as a basis for automotive software testing, are (Roßner, 2010):

- Component Test

- Integration Test

- System Test

\section{- Acceptance Test}

Sensor signal simulation at Component Tests or so called Unit Tests are quiet simple. For example, with a simulation of the remaining bus or stimulating physical hardware signals via e.g., pulse-width modulation (PWM) generators. If we look at the Integration Test or System Test, the distribution of functions increases complexity. In addition simulation of input signals is getting more and more complicated.

In the following, the Navigation system (Navi) consisting of two components is used to explain the developed method of integrated signal virtualisation. The structure symbol in Figure 3 symbolizes the structure of the navigation system menu.

Figure 3 shows the same architecture for both cases and two SWCs representing a distributes system. Figure 3 shows a simplified software architecture with the main layers. From bottom up, above the HW there is the basic software (BSW) allocated. The BSW contains modules shown in Figure 2 primary providing an operating system, communication services, signal- and communication abstraction and HW-driver. All communication to the ApplicationSWCs is distributed via the runtime environment (RTE).

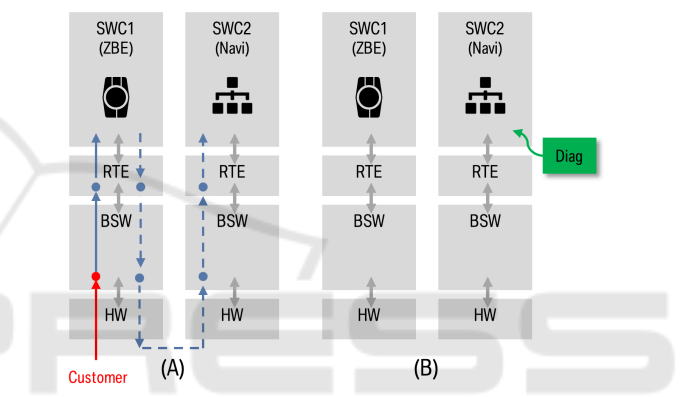

Figure 3: Software-Architecture - [simplified draft]; (A) Customer, (B) Simulation state-of-the-art.

Conventional software-based simulation methods interfere at the application SWC layer, shown in Figure 3(B) named 'Diag'. Usually these functions are integrated for software-based internal error detection and setting data trouble codes (DTC).

Figure 3(A) shows a customer interaction and signal path with the total system via the iDrive controller (ZBE) representing the human machine interface (HMI). The signal path starts at the SWC1 BSW and is handed to SWC1. SWC1 interprets the customer input and sends a message via HW interface to SWC2.

With reference to the example, we look closer to the software architecture at a test case specified in Listing 1. The test case is called 'Start Guidance via entering Navigation Destination' and can be operated by the customer in several ways. This is the reason to restrict the operation to a specific test case. Following manual test is synonymous with customer use.

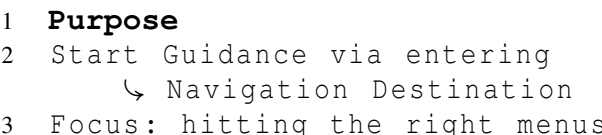




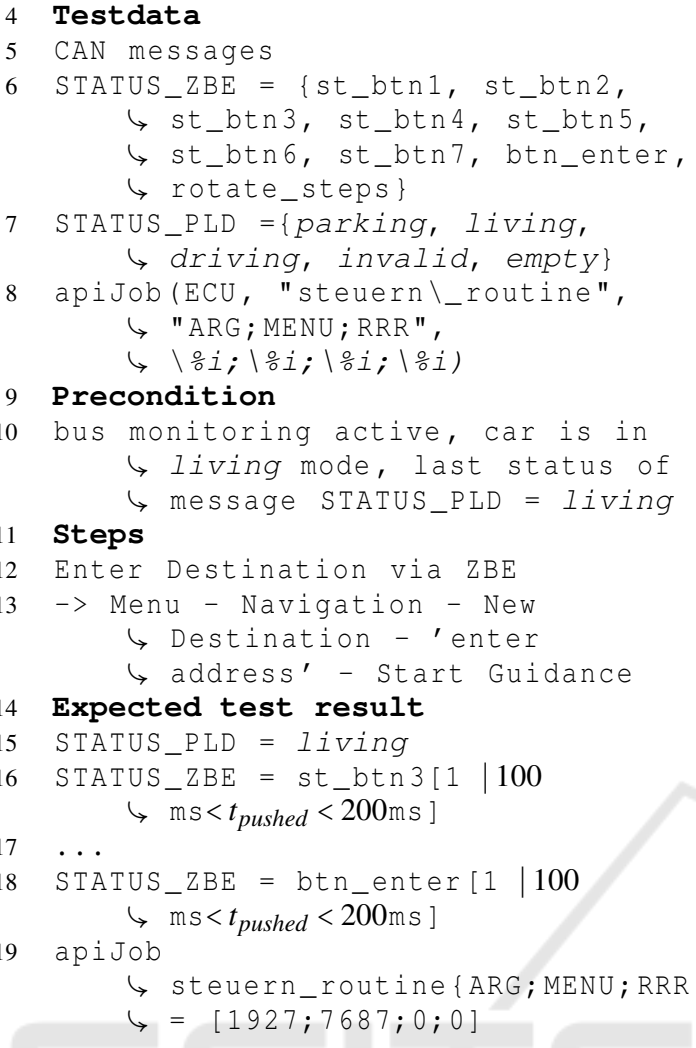

Listing 1: Example test case 'Start Guidance via entering Navigation Destination'.

The conventional virtual interaction uses functions, called diagnosis job (Listing 2) to hit the right menu items.

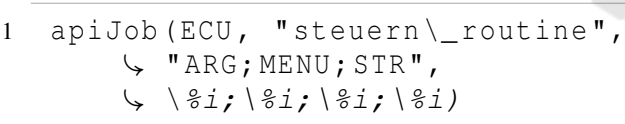

Listing 2: Example Diagnosis-Job.

This function uses a unique identifier (ID) to hit a specific menu item. A series of these jobs is necessary to reach the same goal (in this case an entered navigation destination). Thus the test result for this test has to be Menu ID = 1927 and selected Menu Item $=7687$. For both cases we see the same appearance (MENU_ITEM) shown in Listing 3, but in case of the simulation (Listing 3 - Diag.) the CAN messages are missing.

The test with this kind of customer simulation is far away from the normal customer use (Listing 3 manual). The state-of-the-art simulation method only tests the internal behaviour of a single software component. The result is that errors in the data communication that occur in the customer case are not detected.

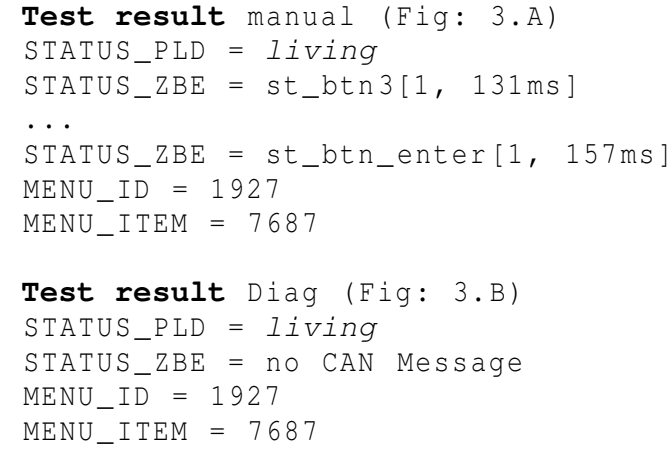

Listing 3: Result test case 'Manual vs. Diag'.

\section{THE APPROACH}

The goal is comparing system- and test model with a new approach via an integrated software-based simulation of input signals. Figure 4 shows the model-based approach of comparing system- and test model. ${ }^{123}$

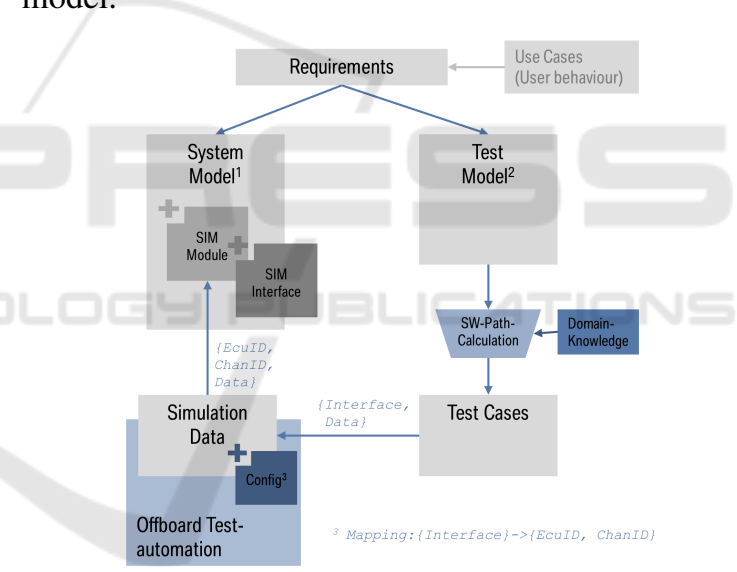

Figure 4: Approach of comparing system- and test model based on a standard model based approach. ${ }^{1,2,3}$

The methodological approach is to integrate a generic simulation module (SIM Module) and a simulation interface (SIM Interface) in the system model on each component. The novelty of this approach is the layer of interaction (AUTOSAR driver layer). The aim of the interaction on this lower software architecture level is to reduce data complexity. This is

\footnotetext{
${ }^{1}$ The system model describes the implemented software solution, including code.

${ }^{2}$ The test model describes the specified implementation for the features and the environment model.

${ }^{3}$ Config describes the hardware specific mapping of Interface to ECU identifier (EcuID) and channel identifier (ChanID).
} 
achieved through an abstraction of the data in the off board automation.

- How does this work? - These SIM moduls can receive test cases from an off board test automation system and execute the test cases, individually or jointly, by order of the off board system.

The data for the test cases is computed out of the test model and transferred in abstract test data and a mapping table (Config). The separation has the advantage of using the test case for different hardware configurations. The separation has the advantage of using the test case for different hardware configurations.
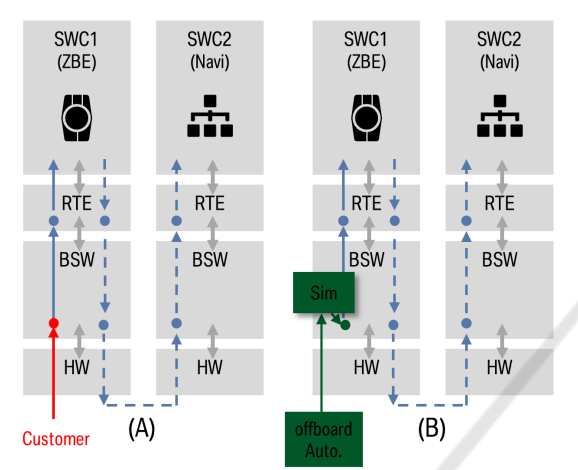

Figure 5: Simulation Approach - SW-Architecture - [simplified draft]; (A) Customer, (B) New simulation approach.

Setting the interaction of the simulation, formerly manipulation, to the BSW driver interface (Figure 5(B)), especially the SWCs operate closer to the customer use. On the one hand, the customer function cycle is closer to the behaviour in the normal customer usage which leads to detection of errors in the functional implementation or errors between layers. On the other hand, the simulation gets easier by getting closer to the hardware layer, because of reduced types of signals. There are only discrete signals, because all data processing of customer input ends up in analog digital converter. A positive effect is that in case of simulation cross-linked functions are triggered as well and show their behaviour as well as the misbehaviour.

\subsection{The Details}

The new methodological approach in Figure 4 shows schematically how the simulation data is loaded to the SIM module. Based on the assumption that a test model is available test cases are derived therefrom. In the derivation of test cases, domain knowledge is added to obtain relevant test cases. This test case data is divided into data sequences for each simulation port and a mapping table. In distributed systems, the use of domain knowledge is meaningful because the functions set conditions and limitation to each other. E.g the power-train domain has information about the specific engine speed range, which is directly linked to the energy availability in the power-network domain. This leads to a reduction of possible test cases.

The bigger part of software functions is developed with a model based approach and realized with state machines. The main reasons for the increase of complexity are on the one hand, that conditions for triggering the transitions are built in various state machines in different software levels. On the other hand almost all of the conditions do have timing constrains. This expands the number of use cases by testing boundary values e.g., lower limit, upper limit, lower limit follower, upper limit follower and last but not least the time steps on the valid timing interval.

Computing the paths of the state management has been performed with a data flow based model analysis (Saad and Bauer, 2013) (Saad and Bauer, 2011). All paths had to start and end in the main menu with the requirement that every transition can be passed only once per path to avoid loops. The result is a set of paths with the information of states passed, transitions and trigger for transitions.

The mentioned mapping table ('Config') is enriched with data from the deployment, with the aim to obtain a architecture-specific mapping table. The deployment is a part of the software development process of AUTOSAR. (AUTOSAR Partnership, 2014)

The configuration ('Config') is an implementation specific information computed out of:

- Software Component Description: Describes the functional dependencies

- System Description: Describes the partitioning of SWCs to ECUs

- ECU Configuration: Describes the signal routing to the HW-Abstraction

- Basic Software Description: Describes the mapping of the HW-Abstraction to the HW-Channels

These descriptions are provided on generating the specific AUTOSAR software configuration for the specified architecture. Therefore the information is depending on the software of the system model. Errors in the AUTOSAR configuration files are handed to the simulation. Faults in the hardware port mapping in the driver layer will not be noticed, only by manual testing of correct connection of the sensors to the ECU (Unit Test).

- The software architecture - Figure 6 shows the software architecture of the integrated simulation approach reduced to its modules and ports. 


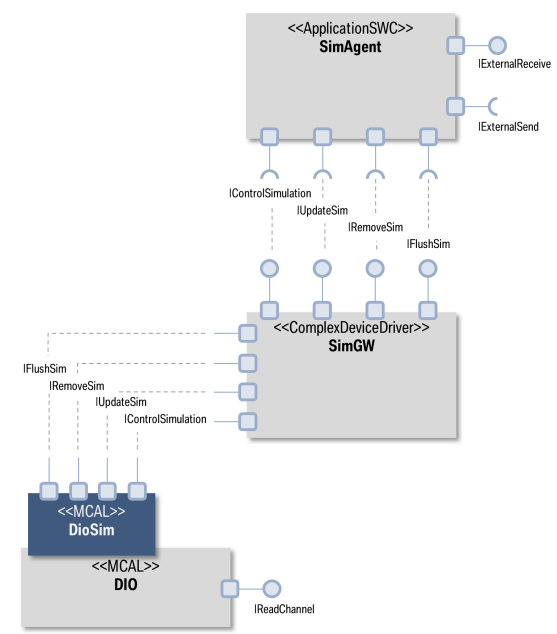

Figure 6: Software Architecture of Simulation module (Köberl, 2015).

The new approach is that the SIM module is integrated in each ECU, which reads sensor signals. The three main components are:

- SimAgent is the logical component, including a state management and is responsible for the execution of the sequences, the internal data storage and all safety requirements,

- $\operatorname{Sim} G W$ is a temporary solution to avoid changes in the hardware abstraction and does only route the signal data to the MCAL-Layer,

- DioSim is the interface to the existing Dio and its read services with the goal to replace the physical signals with the simulated, stored in the SimAgent.

The Gateway module is used to route the signals from the SimAgent to the DioSim. This is a temporary solution that we do not have to edit the I/O-Abstraction of the existing AUTOSAR basic software. Above the RTE there will be the Sim Agent as a part of the BMW System Function Software Components next to the normal application software components (Figure 7). BMW System Functions are standardized software components that are integrated into each ECU, e.g., Diagnosis or DTC-Functions.

The allocation of the modules in the AUTOSAR layered architecture is shown in the Figure 7. Standard SWCs like system time, standard diagnosis, authentication or mentioned above are clustered in the BMW System Function Components.

- The simulation process - Enabling of the simulation will follow the process shown in sequence diagram Figure 8.

- Init(), activating the SimAgent via diagnosis job (CAN-Message),

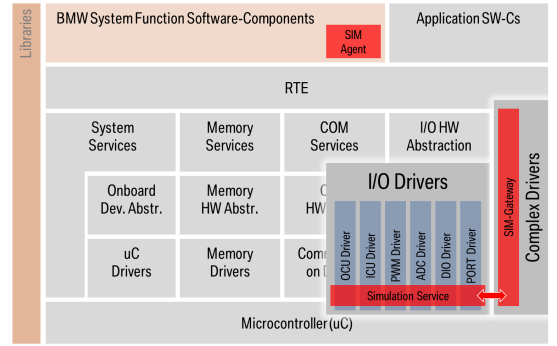

Figure 7: Integration of Simulation Interface in AUTOSAR Driver Layer.

- FlushSim(), erasing existing data in the memory,

- UpdateSim(), setting initial values and parameters, like start value and start time,

- EnableSimulation(), activating the simulation service.

After enabling the simulation service, the $U p$ dateSim-Function is used to feed the DioSim-module with the data during the simulation. The provision of the simulation data in the driver layer is noninteracting with the AUTOSAR read services, in order not to influence the BSW behaviour.

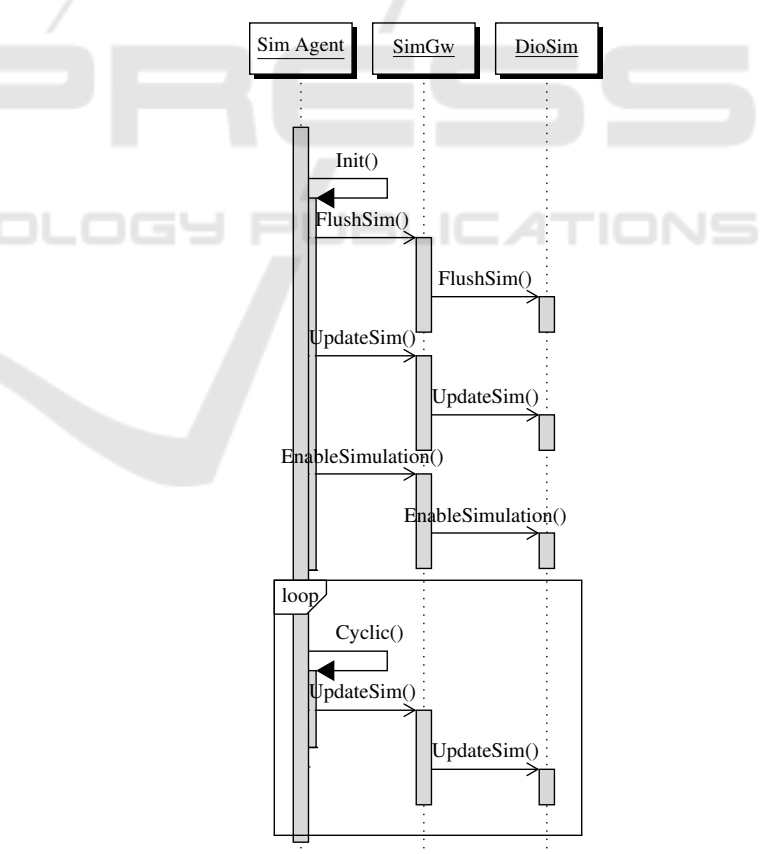

Figure 8: Process Start of Simulation Service (Köberl, 2015).

The novelty here is that a test case, respectively the signal sequences, is saved locally in the ECU memory and is executed by the SIM Agent. Each SIM module has to keep only the information necessary for the ECU specific signal simulation. 
Reading data from the RTE and the chain of reaction through the AUTOSAR basic software is shown in Figure 9. If there is a request of a RTE variable, the normal chain of reaction will be triggered. If the request reaches the Dio-module the Dio-module will check if there is a simulation active for the requested channel (GetSimState()) and will switch to the simulated data if required. In all other cases the physical state of the hardware input port will be read.

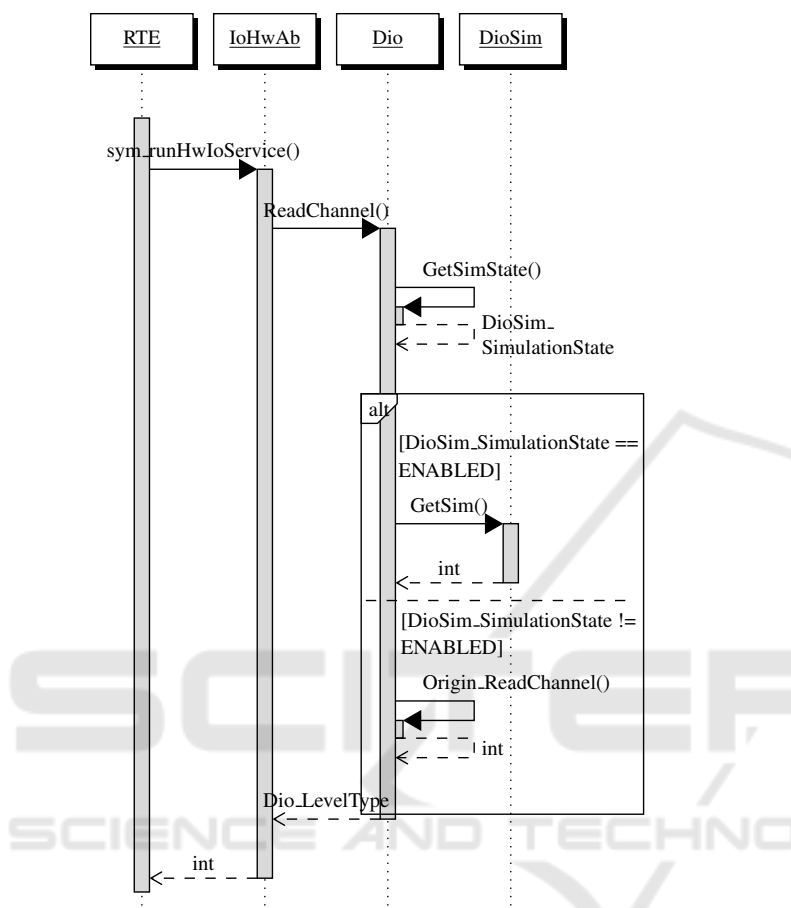

Figure 9: Simulation Service process (Köberl, 2015).

The additional function request GetSimState() will be integrated in the Dio-module and will have an insignificant influence on the time response of the request. There will be no difference between the time response in normal customer use or in simulation usage, because in both cases the new additional function request will be triggered.

The worst case execution time analysis (WCET) calculates an increase of the processor workload about $3,3 \%$. This is a calculation for 255 simultaneously simulated channels, on an $80 \mathrm{MHz}$ CPU with a task cycle of $1 \mathrm{~ms}$ and in case of an error, that no simulation data is available. In this case the software reads, after failure of reading a simulated value, the physical value of the hardware input port.

Based on an $80 \mathrm{MHz}$ CPU, we are using for research and analysis, with a $12.5 \mathrm{~ns}$ Assembler instruction we calculated execution times for $1 \mathrm{~ms}$ tasks. The first one is with no simulation active and the second one is with simulation active without data, representing the worst case scenario.

- DioSim_GetSimState $=0$ [normal case $]$

$\&$ no active Simulation

$\mathrm{WCET}=12,5 \mathrm{~ns}$ according to 1 function call

- DioSim_GetSimState= 1 [worst case]

\& no Simulation Data

$\&$ Reading real data

WCET $=32950 \mathrm{~ns}$

An impact could be a time delay being critical for a SWC. In normal case the SWC runs in tasks about $10 \mathrm{~ms}$ and the calculated delay will be about less than $0,01 \%$.

The last part is a SWC controlling the simulation located on the ECUs to control the simulation process in consideration of safety and CPU-Performance of the ECU and the memory workload. This SWC will be added in further tests to make sure that there will be enough CPU-Performance left. Stopping mechanisms and fall-back solutions are already integrated.

The results of comparing the system reaction in customer usage to the conventional automation approach deviate. The following results (Listing: 4), with the integrated new simulation approach, show a similar system reaction to the customer usage.

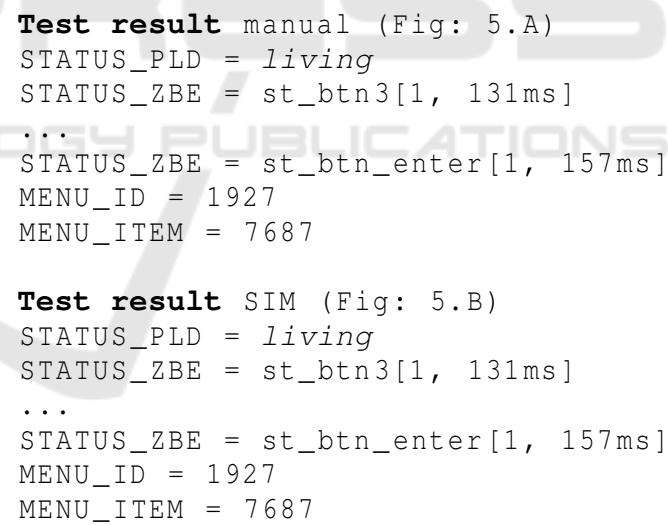

Listing 4: Result test case 'Manual vs. SIM'.

First researches show that the simulation of one to fourteen digital imput ports does work on a virtual ECU without measurable effects on the CPUPerformance.

\subsection{Related Work}

Model based testing is the basic approach in the automotive product development. Methods for data-flow analysis with the help of domain knowledge can help to improve the explained method by computing realistic software-paths and the corresponding data sets. 
With the help of this information, the test model can be improved to get near $100 \%$ test coverage in consideration of the customer use cases. Domain knowledge is the key for computing user realistic paths. Unrealistic paths only have to be checked referring their impact on customer functions and common bugs.

A similar approach with focus of 'automation, modularization and compatibility of all equipment to do measurement, calibration and diagnosis' (ASAP, 1999 ) is the Can Calibration Protocol (CCP). The Protocol is used for calibration and data acquisition. Realized as a driver with access to the internal ECU memory this part of the protocol has a similar effect on the CPU load as the introduced approach. During a session using CCP a 'continuous logical connection' (ASAP, 1999) is established to transfer data from the ECU to the master device (off board test automation).

This approach has a substantial similarity in the connection layer: both interact at the driver layer. However, according to the illustrated approach the CCP has the main goal of data acquisition in contrast to data simulation. With the enormous difference of cutting the data communication to the agent (slave) the new approach is, in case of simulation, way nearer to the customer case.

\section{CONCLUSIONS}

The method of integrated hardware signal simulation, with an integrated software approach, allows to simulate the user input analogous to the customer use cases and thereby to compare system- and test model in an innovative way. The approach shows a different solution with no need of special hardware equipment because of the integration of the simulation in the distributed system as a distributed system. Realized as a standard module for easy integration in the AUTOSAR basic software to get an interface for simulation. The key aspect is the point of interaction located in the AUTOSAR driver layer. The approach uses an abstraction to simplify the data to generic signal sequences as well as to be able to adapt easily to different hardware configurations.

A simple generic simulation module controls the process of simulation, because of its simplicity the simulation does not have a measurable effect on the processor workload. The total system reaction, respectively the system interaction with the environment and customer will be evaluated with proven and tested methods, already in use. Therefore, there is no need in building up new evaluation methods.

Next steps for the simulation approach are to check the data size of the simulation sequences, es- pecially for long term simulations, considering of the limited memory space in automotive ECUs. This fact is the main limitation for the length of data sequences at this time.

\section{REFERENCES}

ASAP (1999). Can calibration protocol - version 2.1.

AUTOSAR Partnership (2014). Autosar components.

Balzert, H. and Ebert, C. (2008). Lehrbuch der Sofwaretechnik. Spektrum Akademischer Verlag, Heidelberg, 2 edition.

Beizer, B. (1990). Software testing techniques. International Thomson Computer Press, New York, 2 edition.

Byhlin, S., Ermedahl Jan Gustafsson, A., and Lisper, B. Applying Static WCET Analysis to Automotive Communication Software.

Eigner, M. and Stelzer, R. (2013). Product-LifecycleManagement. VDI. Springer, Berlin, Heidelberg, 2 edition.

Hoffmann, D. W. (2008). Software-Qualität. EXamen.press. Springer, Berlin, Heidelberg.

Köberl, M. (2015). Integration softwarebasierter automatisierungsmethoden in eine test-ecu. Master's thesis, University of Augsburg, Augsburg.

Myers, G. J., Sandler, C., and Badgett, T. (2012). The art of software testing. John Wiley \& Sons, Hoboken, N.J, 3 edition.

Pezzè, M. and Young, M. (2008). Software testing and analysis. Process, principles, and techniques. Wiley, [Hoboken, N.J.].

Roßner, T. (2010). Basiswissen modellbasierter Test. dpunkt.verl., Heidelberg, 1 edition.

Saad, C. and Bauer, B. (2011). Industry track of software language engineering (itsle), 4th international conference on software language engineering (sle 2011)(may 2011). The Model Analysis Framework An IDE for Static Model Analysis.

Saad, C. and Bauer, B. (2013). Model-driven engineering languages and systems. Data-Flow Based Model Analysis and Its Applications, pages 707-723. Springer.

Seidl, R., Baumgartner, M., and Bucsics, T. (2011). Praxiswissen Testautomatisierung. Basiswissen Testautomatisierung. dPunkt.

Vector Informatik GmbH (2013). Autosar configuration process - how to handle 1000s of parameters. 